\section{Varusschub und Schmerzen im unteren Rückenbereich}

lijima H et al. Relationship Between Varus Thrust

During Gait and Low Back Pain in Individuals

With Knee Osteoarthritis. Arthritis Care Res

2020; 72: 1231-1238

Japanische Orthopäden überprüften, ob ein während des Gehens sichtbarer Varusschub mit einer höheren Prävalenz von Schmerzen im unteren Rückenbereich bei Personen mit Gonarthrose (OA) assoziiert ist.

Die Studie ist eine Sekundäranalyse von Daten einer Querschnittstudie, in der der Zusammenhang zwischen Schmerzen im unteren Rückenbereich (LBP), der Schwere von Schmerzen im Knie und größeren Behinderungen bei Personen mit Gonarthrose untersucht wurde.

Patientinnen und Patienten mit einer Ausprägung der Gonarthrose $\geq 1$ nach dem Kellgren-Lawrence-Score, wurden einer beobachtenden Ganganalyse unterzogen, um den Varusschub zu beurteilen. Schmerzen im unteren Rückenbereich und den Schweregrad bewerteten die Studienteilnehmer mithilfe von Fragebögen. Dabei wurden Frequenz (täglich, fast täglich, einige Tage), Dauer (<3 Monate, 3-7 Monate, 7 Monate -3 Jahre, $\geq 3$ Jahre) und Schwere (anhand einer 10 Punkte Skala) dokumentiert. Analysiert wurde der Zusammenhang zwischen Varusschub und LBP.

Alles in allem wurden 205 Patientinnen und Patienten in die Auswertung eingeschlossen. Das mittlere Alter lag bei 68,19 $\pm 9,23$ Jahren, 72,2\% der Studienteilnehmer war weiblich. Insgesamt 45 Teilnehmer (22,0\%) zeigten einen Varusschub in ihrem schmerzenden Knie, davon 29 bei jedem Schritt und 16 bei einem beliebigen Schritt. Von den 160 Patientinnen und Patienten ohne Varusschub im betroffenen Knie, war in 16 Fällen eine Varusschub im nicht schmerzenden Knie feststellbar.

Diejenigen mit Varusschub hatten häufiger jegliche Art von $\operatorname{LBP}(68,89$ vs. 54,38\%) sowie einen mittelschweren bis schweren LBP, mit einer numerischen Bewertung $\geq 4 / 10$ Punkten (40\% vs. $16,88 \%$ ) im Ver- gleich zu denjenigen ohne Varusschub. Patientinnen und Patienten mit Varusschub hatten ein 3,6-fach höheres Risiko für das Auftreten von mittelschwerem bis schwerem LBP $(95 \%$ KI 1,62-8, 10). Eine Subgruppenanalyse bei insgesamt 118 Studienteilnehmern (31 mit und 87 ohne Varusschub) unter Einbeziehung von Frequenz, Dauer und Intensität der Schmerzen im unteren Rückenbereich, ergab, dass bei Patientinnen und Patienten mit LBP das Vorhandensein von Varusschub mit einer stärkeren LBP-Intensität assoziiert war (proportionale Odds Ratio 2,25 [95\% KI 1,02-4,96]). Einen signifikanten Zusammenhang von Varusschub und LBP-Frequenz sowie Dauer war nicht nachweisbar.

\section{FAZIT}

Die Ergebnisse dieser Analyse unterstreichen erstmalig den Zusammenhang von Varusschub und LBP, was das Konzept einer biomechanischen Verbindung, des sogenannten Knie-WirbelsäulenSyndroms, bekräftigt. Damit liefern diese Daten neue Erkenntnisse zur Klärung der Pathogenese von LBP im Zusammenhang mit einer Gonarthrose, so die wissenschaftliche Arbeitsgruppe.

Richard Kessing, Zeiskam 